\title{
Continuous Reaction Crystallization of Struvite in a DTM Type Crystallizer with Jet Pump of Ascending Suspension Flow in a Mixing Chamber-Kinetic Approach of the Process
}

\author{
Agata Mazienczuk ${ }^{1}$, Andrzej Matynia ${ }^{1}$, Krzysztof Piotrowski ${ }^{2}$, Boguslawa Wierzbowska $^{1}$ \\ ${ }^{1}$ Faculty of Chemistry, Wroclaw University of Technology, Wroclaw, Poland; ${ }^{2}$ Department of Chemical \& Process Engineering, \\ Silesian University of Technology, Gliwice, Poland. \\ Email: krzysztof.piotrowski@polsl.pl
}

Received February $28^{\text {th }}, 2012$; revised April $2^{\text {nd }}, 2012$; accepted April $12^{\text {th }}, 2012$

\begin{abstract}
Reaction crystallization of struvite in water solutions containing 0.20 mass $\%$ of phosphate $(\mathrm{V})$ ions by magnesium and ammonium ions addition was investigated experimentally. Process was carried out in DTM type crystallizer with liquid jet pump device in $298 \mathrm{~K}$ assuming stoichiometric conditions. Struvite crystals of mean size $L_{\mathrm{m}} 5.2-23.0 \mu \mathrm{m}$ were produced depending on $\mathrm{pH}(9-11)$ and mean residence time of suspension in a crystallizer $\tau(900-3600 \mathrm{~s})$. Under these conditions linear growth rate of struvite crystals (SIG MSMPR kinetic model) decreased 2-time with the increase in $\mathrm{pH}$ and 3-time with the elongation of mean residence time of crystal suspension from $7.11 \times 10^{-9} \mathrm{~m} / \mathrm{s}(\mathrm{pH} 9, \tau 900 \mathrm{~s})$ to $1.65 \times 10^{-9} \mathrm{~m} / \mathrm{s}(\mathrm{pH} 11, \tau 3600 \mathrm{~s})$. Nucleation rate varied within the $7.9 \times 10^{8}-1.8 \times 10^{10} 1 /\left(\mathrm{sm}^{3}\right)$ limits. Struvite product of maximal linear size exceeded $100 \mu \mathrm{m}$ with $10 \mathrm{vol} \%$ of $<3 \mu \mathrm{m}$ fraction corresponded to $\mathrm{pH} 9$ and $\tau 3600 \mathrm{~s}$.
\end{abstract}

Keywords: Struvite; Crystal Size Distribution (CSD); Reaction Crystallization Kinetics; Continuous DTM Type Crystallizer; Jet Pump

\section{Introduction}

In supersaturated water solutions of magnesium phosphate $(\mathrm{V})$ in presence of ammonium ions-within the $7<$ $\mathrm{pH}<11$ range and at room temperature- the magnesium ammonium phosphate hexahydrate $\mathrm{MgNH}_{4} \mathrm{PO}_{4} \cdot 6 \mathrm{H}_{2} \mathrm{O}$, struvite, MAP precipitates (solubility product $\mathrm{p} K_{\mathrm{sp}}=9.4$ 13.26) $[1,2]$. Both course and final results of this process are mainly affected by: $\mathrm{pH}$, working supersaturation level, temperature, presence of dissolved and solid impurities, as well as mixing intensity and crystallizer construction [3-6]. In modern processes of phosphorus recycling controlled reaction crystallization of struvite is an effect of contacting the magnesium and ammonium ions (e.g. water solution of magnesium chloride and some ammonium salt) with preliminary purified secondary phosphorus resources, covering mainly municipal, industrial liquid wastes or liquid manure rich in phosphate $(\mathrm{V})$ ions [3]. Liquid wastes purification can be thus closely integrated with simultaneous production of crystalline MAP - for direct use e.g. in agricultural industry as a mineral fertilizer slowly releasing its nutrient contents [7].

Effectiveness of struvite reaction crystallization proc- ess is dependent on crystallizer type and its working mode, appropriate arrangement of its interior, location of the reagents inlet ports, and correct selection of its steady-state parameters [8]. In most of DTM (Draft Tube Magma) type crystallizers adjustment of working supersaturation level is reached by induction of controlled circulation of suspension [9]. Magma flow produces homogenisation of temperature and concentrations, suspends the formed and growing crystals, as well as prevents agglomeration. Mechanical device responsible for internal flow of suspension is usually stirrer or circulation pump. Considerably simpler device, nevertheless still not frequently used in practice, is jet pump $[10,11]$. Unique hydraulic arrangement of a jet pump system provides intensive circulation of suspension, thus its good intrinsic mixing without generation of excessive tensions, influencing favourably the mass and heat transfer processes in a crystallizer. Construction of this apparatus makes removal of suspension of representative crystal size distribution (CSD) possible. It can be assumed, that DTM type crystallizer with jet pump fulfils, at least roughly, the theoretical requirements of MSMPR (Mixed Suspension Mixed Product Removal) crystallizer model 
[8]. This makes estimation of fundamental kinetic parameter values of the process based on product's CSD possible [12].

Experimental results concerning reaction crystallization of struvite from diluted water solutions of phosphate $(\mathrm{V})$ ions in a continuous DTM type crystallizer with internal circulation of suspension driven by liquid jet pump providing ascending flow of suspension in a mixing chamber are presented [5,13]. From the product CSDs fundamental process kinetic parameter values were estimated. The most simplified model of a continuous mass crystallization kinetics in MSMPR crystallizerSIG (Size Independent Growth) model-assuming linear growth rate of all crystals constant regardless their individual sizes, was applied [12].

\section{Experimental}

\subsection{Setup and Procedure}

Scheme of the measurement stand designed for the experiments covering reaction crystallization of struvite is presented in Figure 1(a).

Automatic control and acquisition of measurement data was done by PC computer. Liquid jet pump DTM type crystallizer used in the experiments was a cylindrical tank of $D_{\mathrm{c}} 90 \mathrm{~mm}$ diameter and $H_{\mathrm{c}} 200 \mathrm{~mm}$ height, made of transparent Plexiglas. Diameter of a cylindrical part of the tank overflow was $D_{\mathrm{p}} 120 \mathrm{~mm}$, its height was $H_{\mathrm{p}} 150 \mathrm{~mm}$. Total height of the whole crystallizer was $H_{\mathrm{t}}$ $330 \mathrm{~mm}$, its working part's only was $H_{\mathrm{w}} 220 \mathrm{~mm}$. Crys- tallizer's working volume was $V_{\mathrm{w}} 1.2 \mathrm{dm}^{3}$. Liquid jet pump device (Figure 1(b)) characterized by the following dimensions: mixing chamber diameter $d_{\mathrm{k}} 15 \mathrm{~mm}$; mixing chamber length $l_{\mathrm{k}} 125 \mathrm{~mm}$; confusor diameter (inlet) $d_{\mathrm{o}} 30 \mathrm{~mm}$; confusor length $l_{\mathrm{o}} 12 \mathrm{~mm}$; feeding nozzle diameter (outlet) $d_{\mathrm{e}} 2.0 \mathrm{~mm}$; distance between the feeding nozzle outlet and crystallizer bottom $h_{\mathrm{o}} 25 \mathrm{~mm}$, and distance between feeding nozzle outlet and mixing chamber inlet $b 0 \mathrm{~mm}$.

Crystallizer was continuously provided with a feed: 0.20 mass \% of phosphate $(\mathrm{V})$ ions and - in stoichiometric proportions-with other ions: $\left[\mathrm{Mg}^{2+}\right]_{\mathrm{RM}}=0.0512$ and $\left[\mathrm{NH}_{4}^{+}\right]_{\mathrm{RM}}=0.0380$ mass $\%$. Initially blended reagent solutions were introduced into the process environment as ammonium dihydrogenphosphate $(\mathrm{V}) \mathrm{NH}_{4} \mathrm{H}_{2} \mathrm{PO}_{4}$, magnesium chloride $\mathrm{MgCl}_{2} \cdot 6 \mathrm{H}_{2} \mathrm{O}$ (both p.a., POCh, Gliwice, Poland) and deionized water (Barnstead-NANOpure DIamond). Process temperature was $(298 \pm 0.2) \mathrm{K}$, while environment's $\mathrm{pH}$ was adjusted with water solution of $\mathrm{NaOH}(5 \mathrm{mass} \%)$ to 9,10 and $11( \pm 0.1)$, depending on the current test assumptions. Mean residence time $\tau$ of suspension in a crystallizer working volume varied from 900 to $3600 \mathrm{~s}( \pm 20 \mathrm{~s})$. Process temperature, $\mathrm{pH}$ and inlet/outlet flows (feed solution/product crystal magma) were strictly controlled with PC computer driven by IKA Labworldsoft software. For effective enough internal circulation of suspension, minimal value of unit power of a jet pump feed, $P_{\text {eu }}=0.15 \mathrm{~W} / \mathrm{kg}$, was assumed [13]. Feeding nozzle diameter enabled one to use a possibly minimal feed value, however sufficient enough for continuous

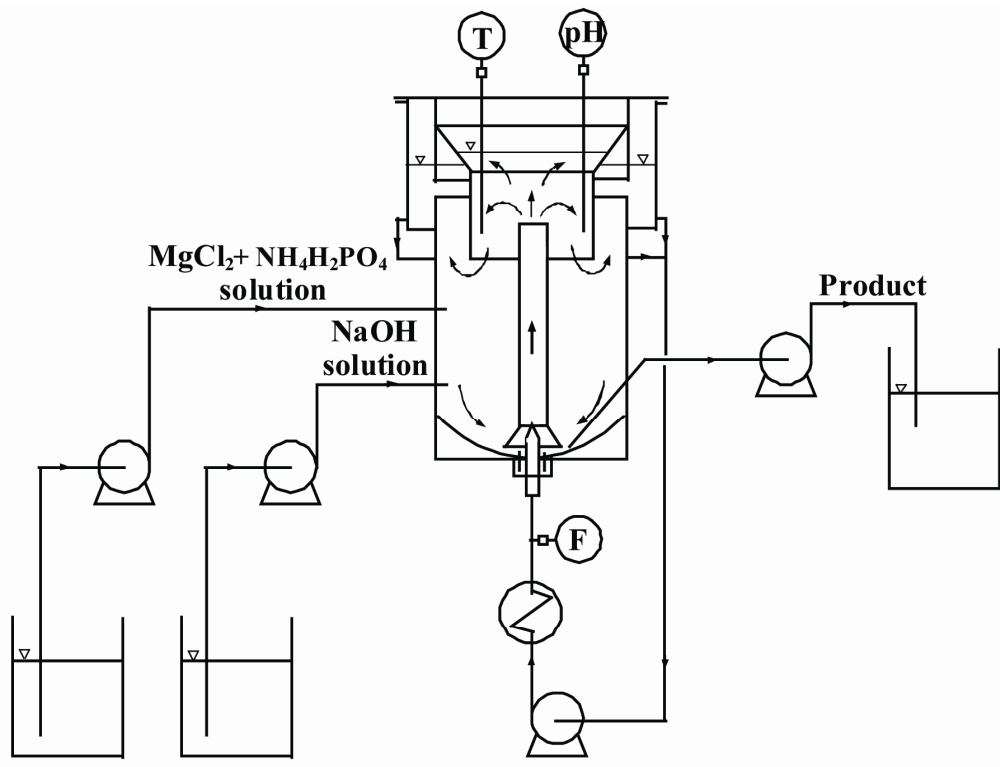

(a)

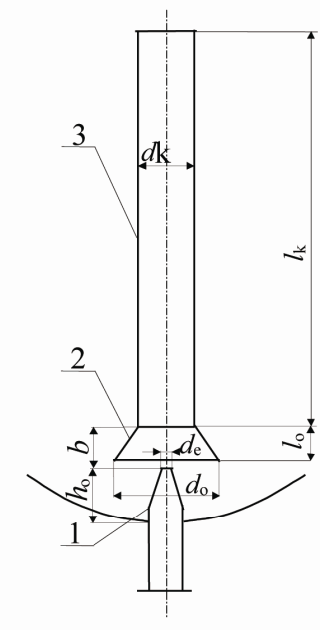

(b)

Figure 1. General scheme of experimental stand (a) and technical details of a jet pump element in the DTM crystallizer; (b) 1 -feeding nozzle, 2 - confusor, 3 -mixing chamber. 
and stable circulation of solid particles in the crystallizer. Intensity of internal circulation was thus also minimal, what advantageously confined attrition and breakage effects. Reagents mixture and $\mathrm{pH}$ correction agent inlets, as well as product suspension outlet are marked in Figure $1(a)$.

Continuous reaction crystallization of struvite ran through $5 \tau$ (starting from the moment of stabilization of the assumed steady-state process parameter values). Then whole crystallizer content was transferred into vacuum filter for mother liquor drainage. The crystals were weighed before and after drying without washing. Mass and volume of mother solution were determined analyticcally. This way solid phase concentration in a product crystal suspension $\left(M_{\mathrm{T}}\right)$ could be calculated. Product CSD was determined with solid particle analyzer COULTER LS-230, whereas their habits were evaluated from scanning electron microscope JEOL JSM 5800LV images. Chemical composition of mother solution and solid phase were determined applying, among others, plasma emission spectrometer ICP-AES CPU 7000, spectrometer IR PU9712, and spectrophotometer UV-VIS Evolution 300. General accuracy of measurement data collected in a continuous laboratory plant was estimated to be $10 \%$ 15\%. Thus in Table 1 mean values of $M_{\mathrm{T}}$ and phosphate $(\mathrm{V})$ ions concentration in a postprocessed mother solution are provided.

Table 1. Influence of selected technological parameters of struvite reaction crystallization process in a continuous DTM crystallizer with a liquid jet pump on the crystal product properties.

\begin{tabular}{cccccc}
\hline & \multirow{2}{*}{ No. } & \multicolumn{3}{c}{ Process parameters } & \multicolumn{3}{c}{ Crystal product characteristics } \\
\cline { 2 - 6 } & $\mathrm{pH}$ & $\tau, \mathrm{s}$ & $L_{\mathrm{m}}, \mu \mathrm{m}$ & $L_{50}, \mu \mathrm{m}$ & $\mathrm{CV}, \%$ \\
\hline 1 & 9 & 900 & 13.2 & 12.1 & 102.0 \\
2 & 10 & 900 & 13.0 & 9.6 & 102.6 \\
3 & 11 & 900 & 5.2 & 3.3 & 99.3 \\
4 & 9 & 1800 & 21.0 & 18.0 & 93.6 \\
5 & 10 & 1800 & 18.3 & 14.6 & 94.6 \\
6 & 11 & 1800 & 5.6 & 4.0 & 94.3 \\
7 & 9 & 3600 & 23.0 & 18.9 & 89.4 \\
8 & 10 & 3600 & 19.2 & 16.2 & 89.6 \\
9 & 11 & 3600 & 7.9 & 6.1 & 88.8 \\
\hline
\end{tabular}

Reagent concentrations in a feed (RM): $\left[\mathrm{PO}_{4}^{3-}\right]_{\mathrm{RM}}=0.20$ mass $\%,\left[\mathrm{Mg}^{2+}\right]_{\mathrm{RM}}$ $=0.0512 \mathrm{mass} \%$, $\left[\mathrm{NH}_{4}^{+}\right]_{\mathrm{RM}}=0.0380$ mass $\%$; Mean concentration of phosphate $(\mathrm{V})$ ions in a postprocessed (outlet) mother solution: $0.011 \pm 0.002$ mass $\%$; Mean concentration of crystal phase in a product magma, $M_{\mathrm{T}}: 4.80$ $\mathrm{kg}$ of struvite $/ \mathrm{m}^{3}$ of suspension.
Automatically determined with the use of COULTER LS-230 product CSDs were then converted into population density distributions (PDDs) [12]. Individual population density values, $n_{\mathrm{i}}\left(L_{\mathrm{i}}\right)$, were determined based on mass $m(L)$ (or volumetric, $V(L)$ ) size distribution data, Equation (1):

$$
n_{\mathrm{i}}\left(L_{i}\right)=\frac{m_{\mathrm{i}}}{k_{\mathrm{v}} \rho L_{\mathrm{i}}^{3} \Delta L_{\mathrm{i}} V_{\mathrm{w}}}=\frac{V_{\mathrm{i}}}{k_{\mathrm{v}} L_{\mathrm{i}}^{3} \Delta L_{\mathrm{i}} V_{\mathrm{w}}}
$$

Analysis of the experimental data was based on SIG kinetic model and assumption of a continuous MSMPR crystallizer conditions fulfillment, Equation (2) [12]:

$$
n(L)=n_{0} \exp \left(-\frac{L}{G \tau}\right)
$$

Equation (2) enables one to determine the values of fundamental kinetic parameters of a continuous mass (reaction-) crystallization process in a theoretically idealized MSMPR crystallizer configuration-nucleation and growth rates. Plot of $\ln n$ versus $L$ gives under these conditions a straight line. Intercept at $L=0$ equals to $\ln n_{0}$ and slope is interpreted as $-1 / G \tau$. If the mean residence time of suspension, $\tau$, can be experimentally adjusted with satisfactory accuracy (e.g. by computer control system), linear growth rate of crystals, $G$, can be calculated directly.

From the nuclei population density, $n_{0}$, and linear growth rate, $G$, the value of nucleation rate $B$ can be determined, Equation (3):

$$
B=n_{0} G
$$

\subsection{Results and Discussion}

The experimental data are presented in Table 1. From the table it results, that with the increase in $\mathrm{pH}$ of struvite reaction crystallization environment, mean crystal size decreases. Increase in $\mathrm{pH}$ from 9 to 11 results in ca. 3-time decrease of mean crystal size, $L_{\mathrm{m}}$ (for $\tau 900 \mathrm{~s}: L_{\mathrm{m}}$ $=13.2 \rightarrow 5.2 \mu \mathrm{m}$, for $\tau 3600 \mathrm{~s}: L_{\mathrm{m}}=23.0 \rightarrow 7.9 \mu \mathrm{m}$ ). Increase in $\mathrm{pH}$ corresponds to increase in a population density of struvite nuclei (see Table 2), resulting in shift of mean crystal size towards smaller values.

Two-time elongation of mean residence time of suspension in a crystallizer (from 900 to $1800 \mathrm{~s}$ ) at $\mathrm{pH} 9$ results, that mean crystal size increases by ca. $60 \%: L_{\mathrm{m}}=$ $13.2 \rightarrow 21.0 \mu \mathrm{m}$. Under these conditions working supersaturation lowers (assuming other process parameters constant), thus decrease of nucleation rate $B$ is observed (see Table 2).

Longer mean residence time is also responsible for establishment of more convenient process conditions for the crystal phase growth. With the reduction of supersaturation linear crystal growth rate $G$ also decreases. 
Table 2. Influence of selected technological parameters of struvite reaction crystallization process in a continuous DTM crystallizer with a liquid jet pump on nucleation rate $B$ and crystal linear growth rate $G$-SIG MSMPR kinetic model applied.

\begin{tabular}{|c|c|c|c|c|}
\hline \multirow{2}{*}{${ }^{1}$ No } & \multicolumn{4}{|c|}{ Process kinetic parameters (SIG MSMPR model) } \\
\hline & $n(L)$ for $L$ & $R^{2}$ & $G, \mathrm{~m} / \mathrm{s}$ & $\begin{array}{c}B, \\
1 /\left(\mathrm{sm}^{3}\right) \\
\end{array}$ \\
\hline 1 & $=1688 \times 10^{17} \exp (-156 ?$ & & $7.11 \times$ & \\
\hline 2 & $=1.066 \times 10$ & 0.996 & & \\
\hline 3 & & & & \\
\hline 4 & $n=1$ & 0.998 & & $10^{8}$ \\
\hline 5 & $n=2.545 \times 1$ & 0.994 & & $10^{8}$ \\
\hline 6 & $n=9.048 \times 10^{17} \mathrm{ex}$ & 0.996 & & \\
\hline 7 & $n=2.801 \times 1$ & 0.998 & & $7.9 \times 10^{8}$ \\
\hline 8 & $n=3.868 \times 10$ & 0.996 & $2.12 \times$ & $8.2 \times 10^{8}$ \\
\hline 9 & $n=5.697 \times 10^{17} \exp \left(-1.683 \times 10^{5} L\right)$ & 0.998 & $1.65 \times 10^{-9}$ & $9.4 \times 10^{8}$ \\
\hline
\end{tabular}

${ }^{1}$ See Table 1.

Elongation of mean residence time of crystals, however, compensates this decrement making longer and more stable growth process possible. It is also responsible for, especially at longer mean residence times, increase in attrition and breakage intensity within larger crystals (for $\tau 3600 \mathrm{~s}$ at $\mathrm{pH} 9: L_{\mathrm{m}}=23.0 \mu \mathrm{m}$, thus increase by ca. $10 \%$ only compared to $L_{\mathrm{m}}=21.0 \mu \mathrm{m}$ for $\tau 1800 \mathrm{~s}$ ). At higher $\mathrm{pH}$ significantly smaller increments in particle sizes with the elongation of mean residence time were observed (see Table 1). Elongation of $\tau$ from 900 to $1800 \mathrm{~s}$ produced at $\mathrm{pH} 10$ increase in $L_{\mathrm{m}}$ by ca. $40 \%$, while at $\mathrm{pH} 11$ -less than $8 \%$ (see Table 2).

Simultaneous influence of process environment's $\mathrm{pH}$ within the $9-11$ range and mean residence time of suspension in a crystallizer $(900<\tau<3600 \mathrm{~s})$ on struvite mean crystal size $L_{\mathrm{m}}$ (in $\mu \mathrm{m}$ ) can be presented in a form of empirical correlation, Equation (4):

$$
L_{\mathrm{m}}=2.86 \times 10^{5} \mathrm{pH}^{-5.44} \tau^{0.328}
$$

of $R^{2}=0.808$ and mean relative error $\pm 25.8 \%$. Graphical projection of this dependence is presented in Figure 2.

Struvite crystals of strongly diversified sizes were removed from the crystallizer. As it results from the data presented in Table 1, coefficient of crystal size variation $\mathrm{CV}$ demonstrated large values, concentrated however within a relatively narrow range: $88.8 \%-102.6 \%$. No unequivocal empirical relation between $\mathrm{CV}$ of crystal product and process parameters could be determined. It can be assumed, that it is a complex, net effect of simultaneous action of $\mathrm{pH}$ and mean residence time of suspension, complemented by attrition and breakage effects on working supersaturation level. Other, random factors can also influence the measurement data precision, including the errors during analyses of dried product CSDs and

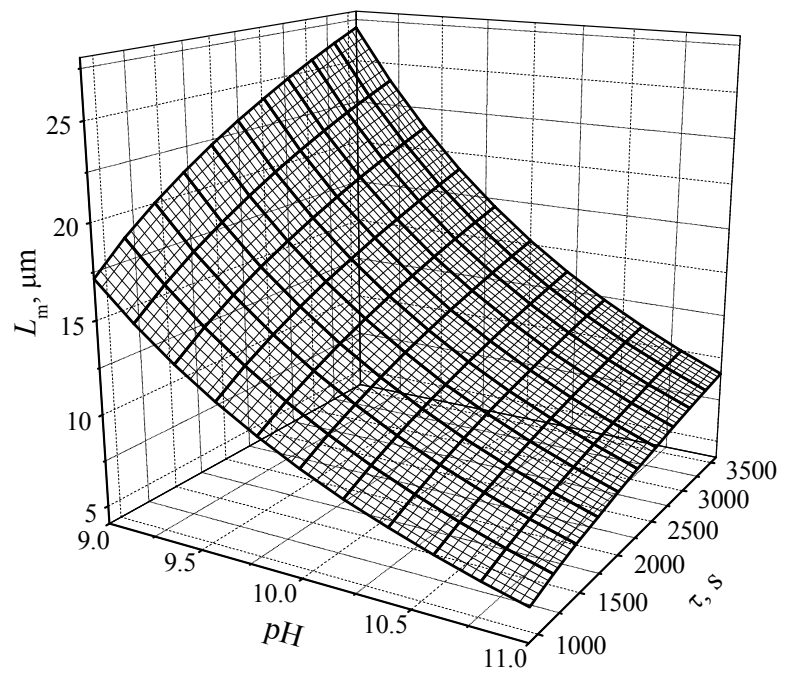

Figure 2. Simultaneous influence of $\mathbf{p H}$ and mean residence time $\tau$ of suspension in a continuous DTM MSMPR crystallizer with a liquid jet pump on the mean crystal size $L_{m}$ of struvite, Equation (4). Process temperature $298 \mathrm{~K}$, concentration of phosphate( $\mathrm{V})$ ions in a feed: 0.20 mass $\%$.

unavoidable oscillations of crystallizer working parameter values $(T \pm 0.2 \mathrm{~K}, \mathrm{pH} \pm 0.1, \tau \pm 20 \mathrm{~s})$. Both crystallizer construction and its work regime make, that limited fraction of the smallest-size struvite crystals reaches the external circulation loop and further circulation pump interior, where these are the subject of additional, more intensive attrition and breakage, thus not representing the hydrodynamic effects inside the crystallizer working volume only. In result crystal products of lower homogeneity are produced, demonstrating smaller mean crystal sizes compared to the products attributed to other types of crystallizer with internal circulation of suspension $[4,6]$. To possibly restrict this phenomenon, minimal value of unit power of a jet pump feeding stream was assumed in the experiments $\left(P_{\mathrm{eu}}=0.15 \mathrm{~W} / \mathrm{kg}\right)$.

In Figure 3 there are presented some exemplary volumetric (mass) struvite product CSDs corresponding to $\mathrm{pH}$ 9, 10 and 11 -all for $\tau 3600 \mathrm{~s}$. With the increase in process environment's $\mathrm{pH}$ dominant crystal size $\left(L_{\mathrm{d}}\right.$, corresponding to maximum of differential distribution) shifts towards smaller sizes: $26.1 \mu \mathrm{m}(\mathrm{pH} 9), 14.9 \mu \mathrm{m}(\mathrm{pH} 10)$ and $6.4 \mu \mathrm{m}(\mathrm{pH} \mathrm{11).} \mathrm{Under} \mathrm{these} \mathrm{conditions} \mathrm{both} \mathrm{number}$ and size of the largest crystals decrease. The largest size of struvite crystals produced at $\mathrm{pH} 9$ is $100 \mu \mathrm{m}$ (Figure 3(a)), at $\mathrm{pH} 10-85 \mu \mathrm{m}$ (Figure 3(b)), while at $\mathrm{pH} 11$ it is only $60 \mu \mathrm{m}$ (Figure 3(c)). Simultaneously fractions of the smallest-size particles increase. In the crystal population produced at $\mathrm{pH} 9$ fraction of sizes below $3 \mu \mathrm{m}$ was $10.1 \%$, while at $\mathrm{pH} 11$ it raised up to $34.7 \%$, thus increased more than 3-time. In effect mean crystal size $L_{\mathrm{m}}$ decreased significantly—from 23.0 to $7.9 \mu \mathrm{m}$ (see Table 1). 
In Figure 4 there are presented selected scanning electron microscope images of struvite crystals which CSDs are shown in Figure 3. Smaller particle sizes corresponding to higher $\mathrm{pH}$ are clearly visible. From the analysis of struvite crystal images it also results, that with the increase in $\mathrm{pH}$ struvite crystals become thinner.

Also their agglomeration degree is higher. Crystal attrition and breakage during mixing and circulation in a crystallizer can be regarded moderate. Only single broken crystals demonstrating some surface destruction effects and rounded edges were observed, however their number was not too large. It speaks advantageously about process conditions established in this crystallizer construction for struvite nucleation and its crystals growth.

In Figure 5 there are presented the experimental po-

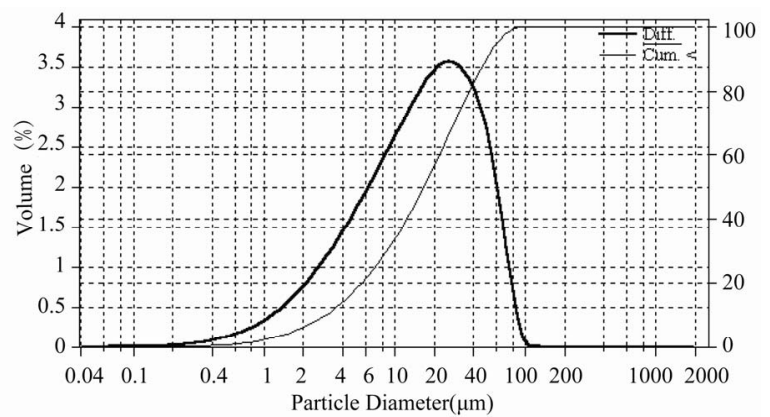

(a)

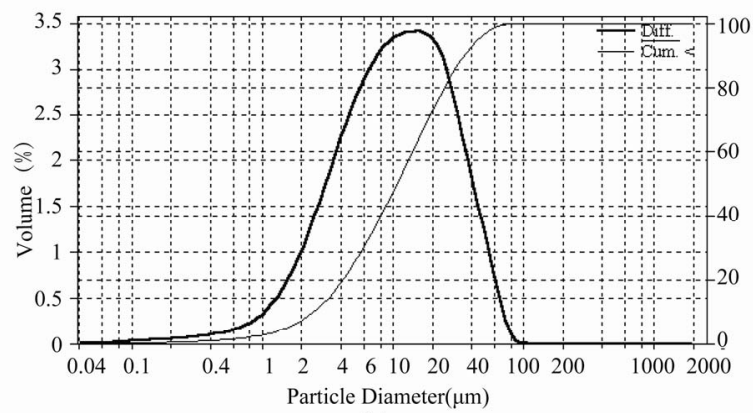

(b)

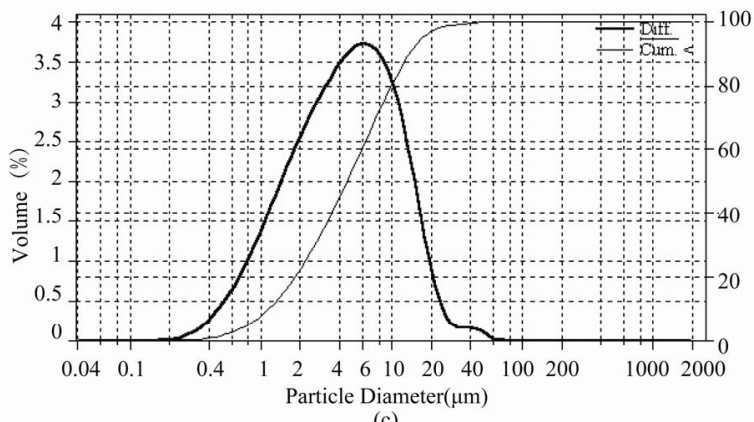

(c)

Figure 3. Exemplary differential (left scale) and cumulative (right scale) volumetric (mass) size distributions of struvite crystals produced in a continuous DTM MSMPR crystallizer with a liquid jet pump for mean residence time $\tau \mathbf{3 6 0 0}$ s: (a) pH 9; (b) pH 10; (c) pH 11-tests No. 7, 8 and 9, respectively, cf. Table 1.

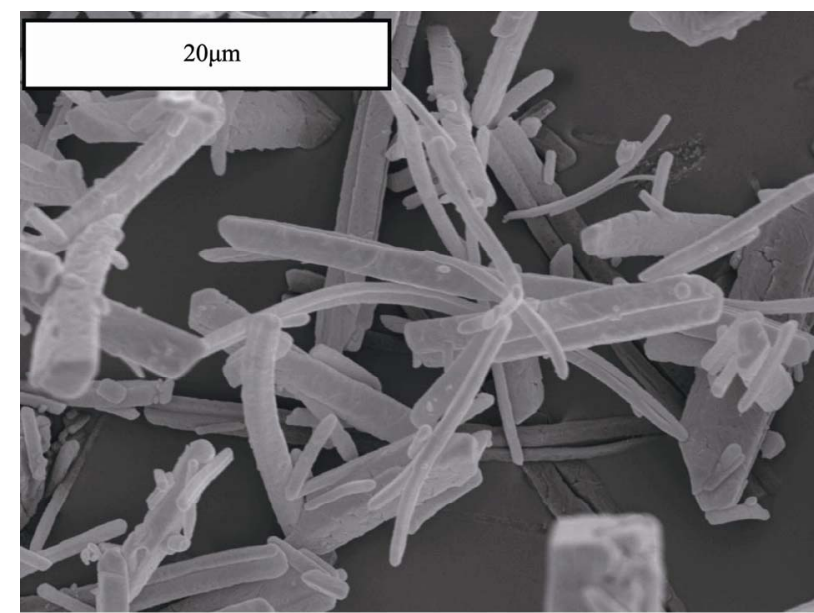

(a)

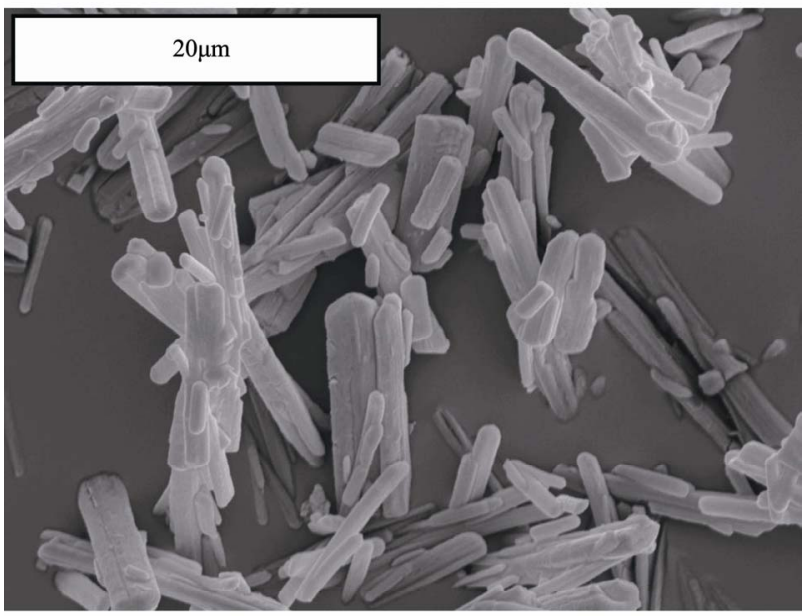

(b)

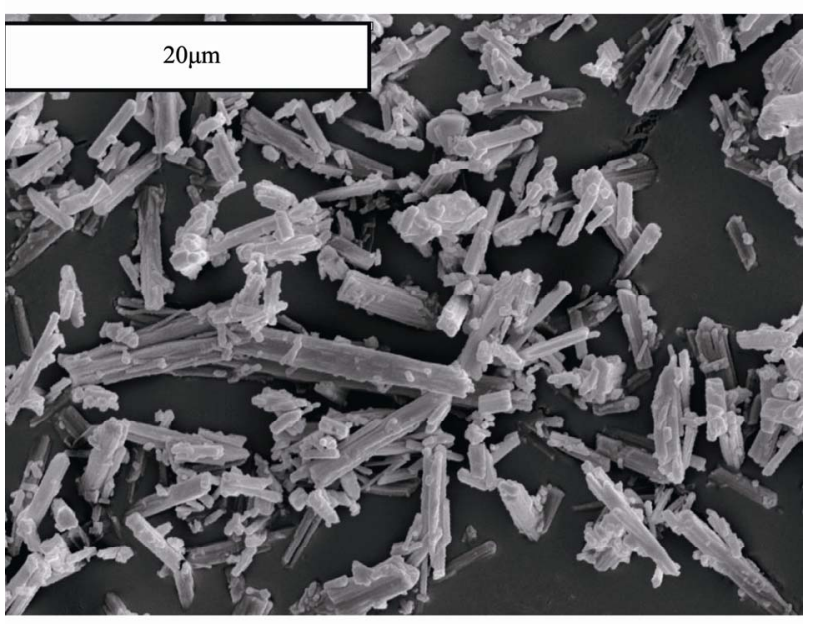

(c)

Figure 4. Exemplary scanning electron microscope images of struvite crystals produced in DTM MSMPR crystallizer with a liquid jet pump for $\tau 3600 \mathrm{~s}$ : (a) $L_{\mathrm{m}}=23.0 \mu \mathrm{m}$ (pH 9); (b) $L_{\mathrm{m}}=19.2 \mu \mathrm{m}$ (pH 10); (c) $L_{\mathrm{m}}=7.9 \mu \mathrm{m}$ (pH 11), magnification $3000 \times$, cf. Figure 3 for size distribution details. 
pulation density distributions (PDDs) of struvite crystals produced at $\mathrm{pH}$ : 9, 10 and 11 (all for $\tau 3600 \mathrm{~s}$ ). Individual population density values $n_{\mathrm{i}}\left(L_{\mathrm{i}}\right)$ were calculated with Equation (1) assuming crystal volumetric shape factor $k_{\mathrm{v}}$ $=1$. From these distribution courses, presented in $\ln n-L$ coordinate system, it results, that for struvite particles of size $L>20 \mu \mathrm{m}$ these dependencies can be with satisfactory accuracy approximated with linear function. Thus from Equation (2) after linear regression one can determine linear crystals growth rate $G$, while from Equation (3) - nucleation rate, $B$. Detailed PDD functions $n(L)$ (Equation (2)) and calculated $G$ and $B$ values are presented in Table 2.

From Figure 5 it also results, that for struvite crystals of sizes $L<20 \mu \mathrm{m}$ these population density distributions demonstrate significant nonlinearity in $\ln n-L$ coordinate system. This characteristic, curved shape can be theoretically interpreted as the occurrence of more complex kinetics of crystal phase growth than it results from preliminary assumed, the most simplified SIG kinetic model $[8,12]$. Theoretical analysis of this phenomenon will be presented in a next authors' work.

Analysing the kinetic data presented in Table 2 one can observe decrease of linear crystal growth rate $G$ with the increase in $\mathrm{pH}$ and with the elongation of mean residence time. In general, higher values of crystal growth rate are observed for shorter mean residence times in an apparatus, what is in accordance with the observations concerning classical, continuous mass crystallization processes [8-10]. Mean value of $G$ for $\tau=900 \mathrm{~s}$ is $5.7 \times 10^{-9}$ $\mathrm{m} / \mathrm{s}$, and for $\tau=3600 \mathrm{~s}-$ only $2.2 \times 10^{-9} \mathrm{~m} / \mathrm{s}$. In turn, relative decrease of crystal growth rate produced by increase in $\mathrm{pH}$ from 9 to $11(\tau=900 \mathrm{~s})$ is $45 \%$. It is not an advantageous phenomenon. Significant decrease of $G$ results in production of crystal population of significantly smaller sizes (Table 1). Simultaneous influence of $\mathrm{pH}$ within the 9 - 11 range and mean residence time of crystal suspension in a crystallizer $(900<\tau<3600 \mathrm{~s})$ on linear growth rate of struvite crystals (in $\mathrm{m} / \mathrm{s}$ ) can be presented in a form of empirical correlation, Equation (5):

$$
G=5.39 \times 10^{-4} \mathrm{pH}^{-2.99} \tau^{-0.683}
$$

with $R^{2}=0.955$ and mean relative error $\pm 10.2 \%$. Graphical projection of Equation (5) is presented in Figure 6.

Nucleation rate $B$, similarly to crystal linear growth rate $G$, decreases with the elongation of mean residence time of suspension in a crystallizer. Elongation of this time value significantly lowers working supersaturation level. For example, elongation of $\tau$ from 900 to $3600 \mathrm{~s}$ results, that nucleation rate decreases from ca. two- to ca. twenty-time, depending on $\mathrm{pH}$. Nevertheless, with the increase in $\mathrm{pH}$ from 9 to 11 nucleation rate, contrary to the crystal growth rate, increases even 15-time ( $\tau 900 \mathrm{~s})$. Higher nucleation rate produces larger fraction of crystal fines, thus smaller product mean size.

In a DTM crystallizer used, compared to DTM variant of descending flow of suspension in a mixing chamber [14], struvite crystals of smaller $L_{\mathrm{m}}$ were produced (by ca. $8 \%$ in average). In the last crystallizer construction feeding nozzle of a jet pump was located below liquid level. Other constructional parameters and continuous work conditions were identical. For the DTM crystallizer variant used in this work also smaller values of linear growth

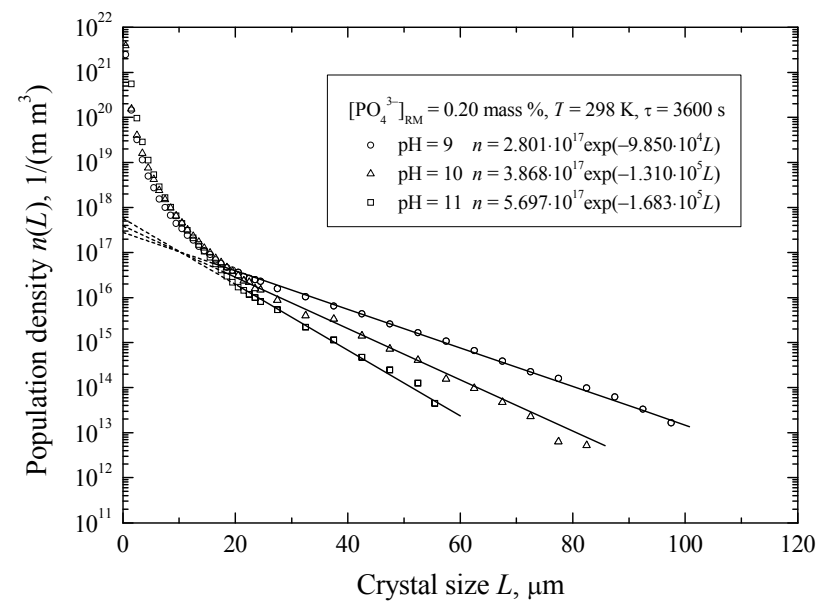

Figure 5. Population density distributions of struvite crystals produced in a continuous DTM crystallizer with a liquid jet pump: the points - experimental data (Equation (1)), solid lines-values calculated with Equation (2) (Table 2) for the crystal sizes $L>20 \mu \mathrm{m}$ (SIG MSMPR kinetic model applied).

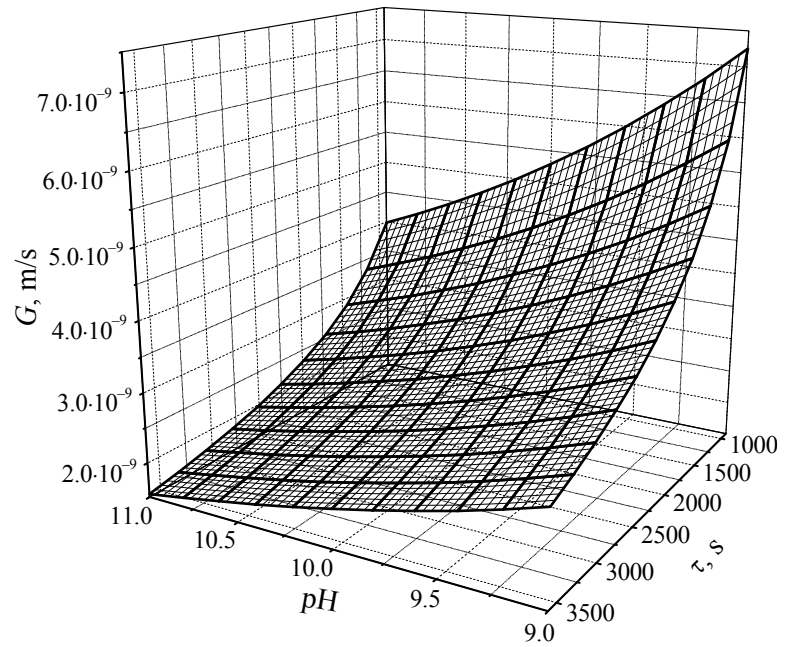

Figure 6. Simultaneous influence of $\mathrm{pH}$ and mean residence time of suspension in a continuous DTM MSMPR crystallizer with a liquid jet pump on linear growth rate of struvite crystals, G, Equation (5). Process temperature $298 \mathrm{~K}$, concentration of phosphate( $\mathrm{V})$ ions in a feed: 0.20 mass \%. 
rate of struvite crystals were observed: for example $G=$ $7.11 \times 10^{-9} \mathrm{~m} / \mathrm{s}(\mathrm{pH}=9, \tau=900 \mathrm{~s})$ and $3.89 \times 10^{-9} \mathrm{~m} / \mathrm{s}$ ( $\mathrm{pH}=11, \tau=900 \mathrm{~s})$ (see Table 2), while in a crystallizer of descending flow these were: $G=7.36 \times 10^{-9} \mathrm{~m} / \mathrm{s}$ and $4.31 \times 10^{-9} \mathrm{~m} / \mathrm{s}[15]$. For all experimental data $G$ was generally lower by nearly $8 \%$ in average. On the other hand calculated struvite nucleation rates were clearly higher: $B=1.2 \times 10^{9}$ and $1.8 \times 10^{10} 1 /\left(\mathrm{sm}^{3}\right)$ (see Table 2) compared to $B=8.1 \times 10^{8}$ and $1.0 \times 10^{9} 1 /\left(\mathrm{sm}^{3}\right)(\mathrm{ac}-$ cording to [15])—for the same parameter values. It can be concluded, that these differences originate mainly from different hydrodynamic regimes. Directions of suspension circulation inside each of these constructions were opposite. Minimal unit power of the jet pump feed stream in a crystallizer of ascending flow was required to be by ca. $70 \%$ higher $\left(P_{\text {eu }}=0.15 \mathrm{~W} / \mathrm{kg}>P_{\text {eu }}=0.09\right.$ $\mathrm{W} / \mathrm{kg}[14,15])$. Fraction of the smallest struvite crystals reaching the crystallizer's overflow and driven further up to a circulation pump interior was ca. 2-time larger, resulting in a more intensified attrition and breakage within these crystals, thus also in a higher secondary nucleation rate.

\section{Conclusions}

From the continuous DTM type crystallizer with liquid jet pump generating ascending flow of suspension in a mixing chamber struvite crystals of mean size $L_{\mathrm{m}}$ from 5.2 to $23.0 \mu \mathrm{m}$ were produced. It was observed, that increase in $\mathrm{pH}$ (from 9 to 11) produced, in average, 3-time decrease of mean crystal size. Contrary, elongation of mean residence time of suspension from 900 to $3600 \mathrm{~s}$ caused significant enlargement of this size (even by more than $70 \%$ at $\mathrm{pH} 9$ ). Crystal products of relatively low size-homogeneity (CV ca. 90\% - 100\%) were removed from the crystallizer. It can be attributed to a complex, net effect of $\mathrm{pH}$ and mean residence time of suspension on working supersaturation level in mother solution, effectively accompanied by attrition and breakage actions within struvite crystals.

Original construction of a liquid jet pump DTM crystallizer (lack of movable elements), its working mode (especially internal circulation of suspension) and low concentration of solid phase in magma $\left(M_{\mathrm{T}} \mathrm{ca} .4 .8 \mathrm{~kg}\right.$ of struvite $/ \mathrm{m}^{3}$ of suspension) did not contribute to excessive attrition and breakage within the crystals. Main source of secondary nucleation was thus circulation pump, provided unintentionally with some amount of the smallest crystals. Analysing all components of a complex continuous struvite reaction crystallization one can notice, that main factor influencing the process course and its results is supersaturation, very strongly dependent (assuming constant: feed solution composition, temperature and suspension mixing/circulation intensity) on $\mathrm{pH}$ and mean residence time of suspension.

For the process kinetic parameters calculation the simplest model valid for ideal MSMPR crystallizer was assumed. It was concluded, that linear growth rate of struvite crystals varied within the $1.65 \times 10^{-9}-7.11 \times 10^{-9}$ $\mathrm{m} / \mathrm{s}$ limits, whereas nucleation rate within the $7.9 \times 10^{8}$ $1.8 \times 10^{10} 1 / \mathrm{sm}^{3}$ range. With the elongation of mean residence time both kinetic parameter values decreased. Raise of $\mathrm{pH}$ resulted in higher nucleation rate and simultaneously lower crystal linear growth rate.

Decrease of $B$ and $G$ with the elongation of $\tau$ is, however, accompanied by increase in mean size $L_{\mathrm{m}}$ of product crystals. Smaller $G$ values are thus compensatedwith excess - by longer contact time of crystals with supersaturated mother solution. Simultaneously decreasing nucleation rates also advantageously influence crystal phase growth and product's CSD. In effect a more convenient conditions of mass transfer between liquid and solid phases are generated, providing additionally more stable crystal growth. In process conditions characterized by relatively long mean residence time of suspension in a crystallizer, higher quality product is formed. However, under such work regime the unit process yield is small, generating thus lower economical effectiveness of a whole production plant.

Raise of struvite reaction crystallization process $\mathrm{pH}$ does not influence the course and results of the process advantageously. This strategy results in higher struvite nucleation rate and decrease of its crystal growth rate. Number of crystal fines in a process system - of lower growth rates-increases, unfavourably modifying the product's CSD. In consequence, at higher $\mathrm{pH}$ levels the corresponding mean size of product crystals reaches only relatively small values.

Concentration of phosphate $(\mathrm{V})$ ions in mother solution, after reaction crystallization of struvite, decreased by ca. $95 \%$, what can be regarded as a very good result of the process of their selective recycling from the inlet solution.

\section{Acknowledgements}

This work was supported by the Ministry of Science and Higher Education of Poland under grant No. NN209 117437 (2009-2012). The work was presented at 18th International Symposium on Industrial Crystallization (ISIC18), ETH Zurich, 13-16 September 2011, Zurich, Switzerland.

\section{REFERENCES}

[1] S. A. Parsons, "Recent Scientific and Technical Developments: Struvite Precipitation," CEEP Scope Newsletters, No. 41, 2001, pp. 15-22. 
[2] J. Doyle and S. A. Parsons, "Struvite Formation, Control and Recovery," Water Research, Vol. 36, No. 16, 2002, pp. 3925-3940. doi:10.1016/S0043-1354(02)00126-4

[3] K. S. Le Corre, E. Valsami-Jones, P. Hobbs and S. A. Parsons, "Phosphorus Recovery from Wastewater by Struvite Crystallization: A Review," Critical Reviews in Environmental Science and Technology, Vol. 39, No. 6, 2009, pp. 433-477. doi:10.1080/10643380701640573

[4] A. Matynia, J. Koralewska, K. Piotrowski and B. Wierzbowska, "The Influence of the Process Parameters on the Struvite Continuous Crystallization Kinetics," Chemical Engineering Communications, Vol. 193, No. 2, 2006, pp. 160-176. doi:10.1080/009864490949008

[5] J. Koralewska, K. Piotrowski, B. Wierzbowska and A. Matynia, "Kinetics of Reaction-Crystallization of Struvite in the Continuous Draft Tube Magma Type Crystallizers -Influence of Different Internal Hydrodynamics," Chinese Journal of Chemical Engineering, Vol. 17, No. 2, 2009, pp. 330-339. doi:10.1016/S1004-9541(08)60212-8

[6] A. Matynia, A. Mazienczuk, B. Wierzbowska, A. Kozik and K. Piotrowski, "New Crystallizers With a Compressed Air-driven Jet Pump in the Process of Precipitating Struvite From Diluted Water Solutions Containing Phosphate Ions," Chemik, Vol. 64, No. 11, 2010, pp. $753-758$ (in Polish).

[7] L. E. de-Bashan and Y. Bashan, "Recent Advances in Removing Phosphorus from Wastewater and Its Future Use as Fertilizer," Water Research, Vol. 38, 2004, pp. 4222-4246. doi:10.1016/j.watres.2004.07.014

[8] J. W. Mullin, "Crystallization," Butterworth-Heinemann, Oxford, 1993
[9] Z. Rojkowski and J. Synowiec, "Crystallization and the Crystallizers," WNT, Warszawa, 1991 (in Polish).

[10] P. Synowiec, "Industrial Crystallization from Solution," WNT, Warszawa, 2008 (in Polish).

[11] A. Matynia, "Crystallizers with a Jet Pump," Inzynieria $i$ Aparatura Chemiczna, Vol. 36, No. 6, 1997, pp. 9-14 (in Polish).

[12] A. D. Randolph and M. A. Larson, "Theory of Particulate Processes: Analysis and Techniques of Continuous Crystallization," 2nd Edition, Academic Press, New York, 1988.

[13] J. Koralewska, K. Piotrowski, B. Wierzbowska and A. Matynia, "Reaction-Crystallization of Struvite in a Continuous Liquid Jet Pump DTM MSMPR Crystallizer with Upward Circulation of Suspension in a Mixing Chamber -An SDG Kinetic Approach," Chemical Engineering \& Technology, Vol. 30, No. 11, 2007, pp. 1576-1583. doi:10.1002/ceat.200700229

[14] J. Koralewska, N. Hutnik, K. Piotrowski, B. Wierzbowska and A. Matynia, "Effect of Reaction Crystallization Parameters on the Quality of Struvite Crystals Produced in a Continuous Draft Tube Magma Crystallizer With a Jet Pump Fed With Recirculated Mother Solution," Przemysl Chemiczny, Vol. 88, No. 5, 2009, pp. 472-476 (in Polish).

[15] J. Koralewska, N. Hutnik, B. Wierzbowska, K. Piotrowski and A. Matynia, "Separation of Crystalline Struvite from Diluted Solutions in a DTM Crystallizer with Liquid Jet Pump of Descending Suspension Flow in a Mixing Chamber," Przemysl Chemiczny, Vol. 89, No. 8, 2010, pp. 1087-1091 (in Polish). 


\section{Nomenclature}

$B$-nucleation rate, $1 /\left(\mathrm{m}^{3} \mathrm{~s}\right)$;

$\mathrm{CV}$ - coefficient of (crystal size) variation, defined as $100\left(L_{84}-L_{16}\right) /\left(2 L_{50}\right), \%$;

$G$-linear growth rate of crystals, $\mathrm{m} / \mathrm{s}$;

$k_{\mathrm{v}}$-volumetric shape factor of crystal;

$K_{\text {sp }}$ - solubility product;

$L$ - characteristic linear size of crystal, $\mathrm{m}$;

$L_{\mathrm{d}}$ - dominant crystal size, m;

$L_{\mathrm{i}}$ - mean size of $i$-th crystal fraction, $\mathrm{m}$;

$L_{\mathrm{m}}$-mean size of crystal population, defined as $\Sigma x_{\mathrm{i}} L_{\mathrm{i}}, \mathrm{m}$;

$L_{50}$ - median crystal size for 50 mass $\%$ undersize fraction, $\mathrm{m}$;

$\Delta L_{\mathrm{i}}$ - size range of $i$-th crystal fraction, $\mathrm{m}$;

$m_{\mathrm{i}}$-mass of $i$-th crystal fraction, $\mathrm{kg}$;

$m(L)$ - crystal mass size distribution;

$M_{\mathrm{T}}$ crystal content in suspension (suspension density), $\mathrm{kg}_{\text {cryst }} / \mathrm{m}^{3}$;

$\left[\mathrm{Mg}^{2+}\right]_{\mathrm{RM}}$ - concentration of magnesium ions in a feed, mass $\%$;

$n_{0}$-nuclei (zero-size crystals) population density, $1 /\left(\mathrm{m} \mathrm{m}^{3}\right)$; $n_{\mathrm{i}}$-population density of $i$-th crystal fraction, $1 /(\mathrm{m}$ $\left.\mathrm{m}^{3}\right)$;

$n(L)$ - population density (number of crystals within the specified size range in unit volume of the suspension per this size range width), $1 /\left(\mathrm{m} \mathrm{m}^{3}\right)$;

$\left[\mathrm{NH}_{4}^{+}\right]_{\mathrm{RM}}$ - concentration of ammonium ions in a feed, mass \%;

$P_{\mathrm{eu}}-$ unit power of a jet pump feeding stream, $\mathrm{W} / \mathrm{kg}$;

$\left[\mathrm{PO}_{4}^{3-}\right]_{\mathrm{RM}}$ - concentration of phosphate $(\mathrm{V})$ ions in a feed, mass $\%$;

$q_{\mathrm{v}}$-volumetric (out)flow rate of crystal suspension from the crystallizer, $\mathrm{m}^{3} / \mathrm{s}$;

$T$ - process temperature, $\mathrm{K}$;

$V_{\mathrm{i}}$-volume of $i$-th crystal fraction, $\mathrm{m}^{3}$;

$V_{\mathrm{w}}$ - crystallizer working volume, $\mathrm{m}^{3}$;

$V(L)$ - crystal volumetric size distribution;

$x_{\mathrm{i}}$ - mass fraction of the crystals of mean fraction size $L_{\mathrm{i}}$.

Greek letters

$\tau$-mean residence time of suspension in a crystallizer working volume, defined as $V_{\mathrm{w}} / q_{\mathrm{v}}, \mathrm{s}$; $\rho$ - density of crystals, $\mathrm{kg} / \mathrm{m}^{3}$. 